\title{
Time Evolution of Ion Energy Distributions for Plasma Doping
}

\author{
Ankur Agarwal and Mark J. Kushner, Fellow, IEEE
}

\begin{abstract}
Plasma doping of semiconductors is being investigated for low-energy ion implantation to form ultra-shallow junctions. Ions are extracted from a quasi-dc plasma using a pulsed bias on the substrate. The shape of the resulting ion energy and angular distribution (IEAD) is particularly important with respect to obtaining desired junction characteristics. Images are presented of the time evolution of the IEAD in a plasma doping system.
\end{abstract}

Index Terms-Ion implantation, modeling, plasma, pulsed.

$\mathbf{U}$ LTRA-SHALLOW junctions (USJ) are required for fabrication of sub- $0.1 \mu \mathrm{m}$ transistors in semiconductor integrated circuits. The most straight forward fabrication method is to extend the beam-line ion implantation technology to ultra-low energies (100s eV to a few kiloelectronvolts). Due to space charge induced divergence, low-energy beams are restricted to low currents resulting in lower throughputs. Several alternative techniques have been proposed for fabricating USJ. The most promising candidates are plasma implantation methods which include pulsed plasma doping $\left(\mathrm{P}^{2} \mathrm{LAD}\right)$ and plasma immersion ion implantation (PIII) [1].

$\mathrm{P}^{2} \mathrm{LAD}$ is an attractive, simple and low cost alternative to beam line technologies. $\mathrm{P}^{2} \mathrm{LAD}$ is capable of delivering high dose rates at ultra-low energies $(0.02-20 \mathrm{kV})$ using conventional plasma processing technologies. In one variation of $\mathrm{P}^{2} \mathrm{LAD}$, a pulsed negative voltage is applied to the substrate to both create a plasma containing the desired dopant species and to accelerate the positive dopant ions the cathode sheath. Another variation uses an inductively coupled plasma (ICP) as the source of ions. Typical bias pulse lengths range between 5 and $50 \mu$ s. For sufficiently low pressures, the ions are implanted into the wafer with energies largely determined by the pulse voltage and the ion charge. The plasma is ignited with each pulse and extinguishes after each pulse ends [2].

In this paper, we present a computationally derived image of the time evolution of the ion energy and angular distribution (IEAD) in a $\mathrm{P}^{2} \mathrm{LAD}$ system as a pulsed bias voltage is applied to the substrate of an ICP. To address these conditions the Plasma Chemistry Monte Carlo Module (PCMCM), inter-

Manuscript received October 10, 2004; revised December 8, 2004. This work was supported in part by the National Science Foundation under Grant CTS0315353 , in part by the Semiconductor Research Corporation, and in part by Varian Semiconductor Equipment Associates.

A. Agarwal is with the Department of Chemical and Biomolecular Engineering, University of Illinois, Urbana, IL 61801 USA (e-mail: aagarw13@uiuc.edu).

M. J. Kushner is with the Department of Electrical and Computer Engineering, Iowa State University, Ames, IA 50011 USA (e-mail: mjk@iastate.edu).

Digital Object Identifier 10.1109/TPS.2005.845887

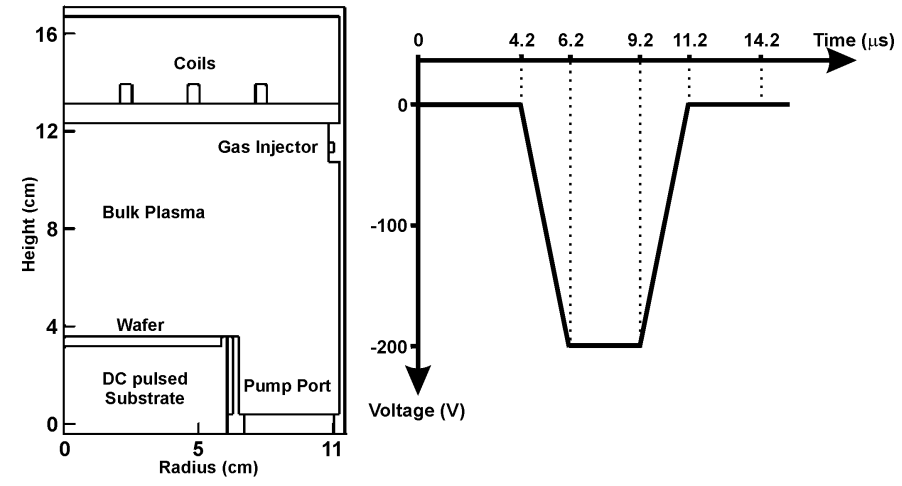

Fig. 1. Schematic of the reactor and pulsed bias voltage waveform.

faced with the Hybrid Plasma Equipment Model (HPEM), was modified to simulate long, nonsinusoidal bias pulses [3]. The HPEM is a two-dimensional $(r, z)$ cylindrically symmetric simulation. It is composed of a series of linked modules which are iterated to a converged solution [4]. The modules used here are: electromagnetics (propagation and power absorption), electron kinetics (electron transport coefficients and source functions), and fluid-kinetics (neutral and charged particle densities, and Poisson's equation for the electric potential).

The model reactor and pulsed bias format are shown in Fig. 1. For demonstration purposes we used argon at 15 mtorr at a flow rate of $100 \mathrm{sccm}$. The ICP power is $300 \mathrm{~W}$ and the wafer was biased at $-200 \mathrm{~V}$ with a $7-\mu$ s-long pulse. The PCMCM produces the time and energy resolved flux of $\mathrm{Ar}^{+}$to the wafer. The fluxes are post-processed to provide the time resolved IEAD. The image was generated from the raw data using Tecplot (v10) [5] and was subsequently annotated using Adobe Photoshop (v 7) [6].

The IEAD during the pulsed bias is shown in Fig. 2. The peak plasma density in the center of the reactor is $1.9 \times 10^{11} \mathrm{~cm}^{-3}$, and at the edge of the sheath prior to bias is $9.8 \times 10^{9} \mathrm{~cm}^{-3}$. Upon applying the bias, the sheath expands to $\approx 0.1 \mathrm{~cm}$, growing in thickness as ions are depleted from the sheath when accelerated into the wafer. The ion density is sufficiently large that the plasma is largely unperturbed in the center of the reactor. For these conditions, the $\mathrm{Ar}^{+}$ions are accelerated collisionlessly across the sheath attaining maximum energies determined by the bias. The time-resolved IEAD is broad $\left( \pm 17^{\circ}\right)$ and of low energy $(30 \mathrm{eV}$, about the unbiased sheath potential) prior and after the pulse. As the bias is applied, the IEAD narrows to its minimum breadth $\left( \pm 8^{\circ}\right)$ when the bias is most negative. Time averaged measurements of the IEAD show multi-energy structures that result from the superposition of these two contributions, one by ions arriving during the pulse-on period (high en- 


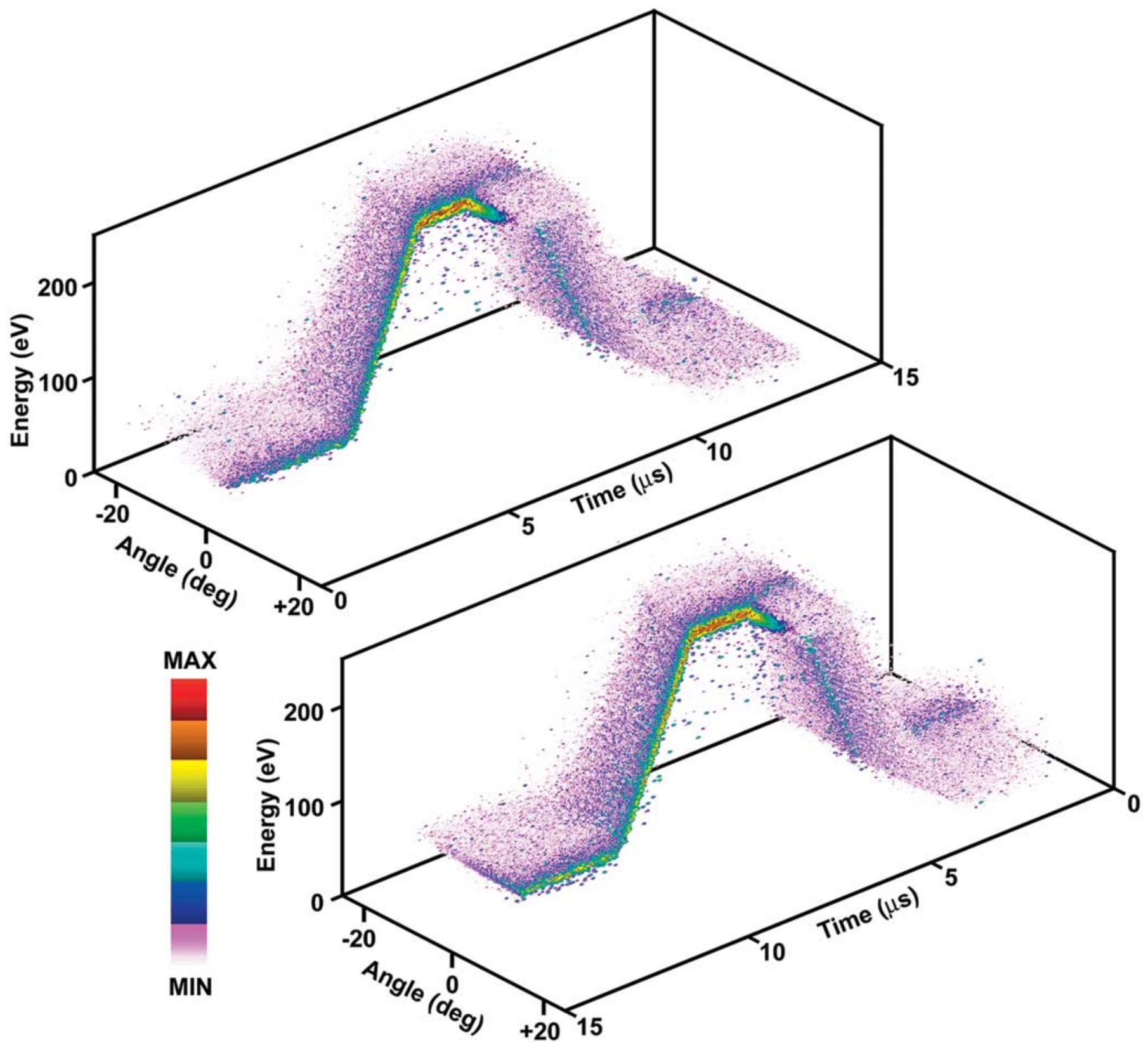

Fig. 2. Time evolution of the IEAD in an ICP with a pulsed dc bias on the substrate. Views are shown from the perspective of early and late times, with cutouts to reveal the high intensity portions of the IEAD. IEAD narrows as the bias voltage decreases to its most negative value.

ergy) and the other by ions arriving during the pulse-off period (low energy). The IEADs can, thus, be tailored to utilize the ions originating from different periods of the pulse to achieve the desired implant characteristics.

\section{REFERENCES}

[1] P. K. Chu, S. Qin, C. Chan, N. W. Cheung, and L. A. Larson, "Plasma immersion ion implantation a fledgling technique for semiconductor processing," Mat. Sci Eng., vol. R17, pp. 207-280, 1996.
[2] S. B. Felch, Z. Fang, B.-W. Koo, R. B. Liebert, S. R. Walther, and D. Hacker, "Plasma doping for the fabrication of ultra-shallow junctions," Surf. Coatings Technol., vol. 156, pp. 229-236, 2002.

[3] A. Sankaran and M. J. Kushner, "Integrated feature scale modeling of plasma processing of porous and solid $\mathrm{SiO} 2$. I. Fluorcarbon etching," $J$. Vac. Sci. Technol. A, vol. 22, pp. 1242-1259, 2004.

[4] R. Kinder and M. J. Kushner, "Non-collisional heating and electron energy distributions in magnetically enhanced inductively coupled and helicon plasma sources," J. Appl. Phys., vol. 90, pp. 3699-3712, 2001.

[5] Amtec Engineering., Bellevue, WA. [Online]. Available: http://www.amtec.com

[6] Adobe Corporation., San Jose, CA. [Online]. Available: http://www.adobe.com 